\title{
Reproducing and Re-experiencing the Writing Process in Japanese Calligraphy
}

\author{
Kumiyo Nakakoji ${ }^{1,2}$, Kazuhiro $\mathrm{Jo}^{1}$, Yasuhiro Yamamoto ${ }^{1}$ \\ Yoshiyuki Nishinaka ${ }^{2}$, Mitsuhiro Asada ${ }^{2}$ \\ ${ }^{1}$ RCAST, University of Tokyo ${ }^{2}$ SRA-KTL Inc., Japan \\ \{kumiyo,jo,yxy\}@kid.rcast.u-tokyo.ac.jp_\{nisinaka,m-asada\}@sra.co.jp
}

\begin{abstract}
Japanese calligraphy is the art of brush writing where a person writes Japanese characters with a Chinese brush against a sheet of paper. We have implemented a mechanism to capture the process of producing Japanese calligraphy using MERL's DiamondTouch (DT) table. We add a very thin metal wire along the length of the brush to carry an electric signal from the writer's body through the brush tuft and ink to the table. As the brush tuft is touches a sheet of paper placed on the surface of the DT table, the ink in the tuft carries the signal from the users to DT. We capture the movement of the brush tuft to produce the visual and auditory representations of the writing process and for later replay.
\end{abstract}

\section{Introduction}

Japanese calligraphy (called "Shodo" in Japanese) is the form of writing Japanese characters with a Shodo brush ("Fude") and ink ("Sumi"). It uses the brush "to deliver harmonious rhythm with varying brush posture, speed, and pressure" [3].

While Japanese calligraphy might be thought of as a visual representation, it is also about the process; its rich visual expressions are the result of dynamic strokes and the rhythm of writing [7]. A stroke's formation "largely depends on the constantly changing brush footprints generated from the artist's manipulation of the brush" [3].

Most children in Japan learn calligraphy in elementary school and some adults adopt it as a hobby. The traditional manner of learning Shodo is to use examples writing as guide for the student to mimic. The example is usually placed to the left of a blank sheet of paper and the student refers to it in practicing with a brush soaked with ink. It is often difficult for the student to match the given example, because it is a static image of a dynamic process. A teacher therefore will sometimes hold the student's brush hand and guide the brush in order to teach the motion, angle, direction and force.

\section{The Basic Idea}

Our approach is (1) to preserve the Shodo experience in a natural setting, and (2) to unveil the temporal and rhythmic process information embedded in the produced visual representation for non-Shodo experts.

We use MERL's DiamondTouch (DT) table [4] to sense the calligraphy activity produced in the natural setting where a user writes with a traditional brush with ink, on a sheet of paper placed on the flat surface of the DT table (Figure 1(a)). Our idea is to use water, more specifically, ink loaded in a brush tuft, as the conductive material between the brush and the DT surface. So we add a very thin metal wire to the brush handle to make an electric circuit from the user's body, through the brush handle, through the ink and finally to the DT surface via the ink flowing onto the paper (Figure 1(b)). Because the brush handle is made of wood, this thin wire is the least obtrusive way to preserve all the most important aspects of the feel of media and medium to the artist.

Because the ink is conductive, we can approximate the touched area of the brush tuft put against the sheet of paper placed on the DT table. Recording a series of the sensed brush touched area allows us to re-produce a Japanese calligraphic representation with temporal information.
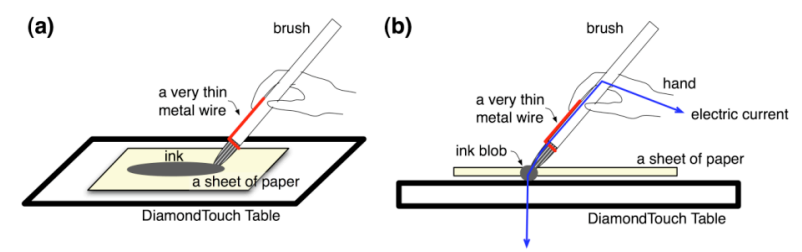

Figure 1: The Mechanism

\section{The Calligramp System}

We have implemented the Calligramp (Calligraphy Amplifier) system based on the above idea.

\subsection{An Overview}

The Calligramp system (Figure 2) detects the brush tuft movements through the DT table and stores them 
as time-stamped stroke data. It then produces visual and auditory representations from the data, and presents them to a user through means such as speakers, a visual monitor, and/or a projector.

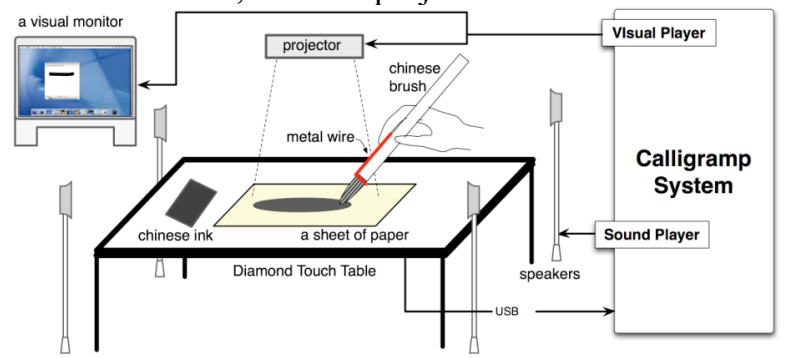

Figure 2: The Calligramp System

Figure 3 shows how a brush tuft touch via a sheet of paper is detected by the DT table. Figure 3-Left shows how our system preserves the physical setting for the user. Figure 3-Right shows how the user's action is sensed in realtime.

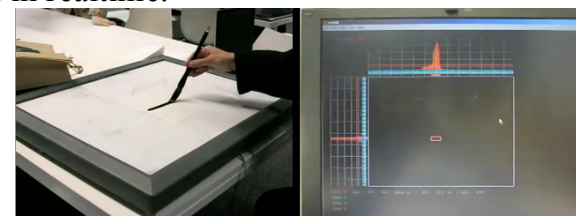

Figure 3: How a Brush Tuft Touch is Sensed by DT

We tested with different types of inks. We originally used black ink, which is usually used in Japanese calligraphy. This ink consists of carbon, which is conductive. The problem is that as the artist adds to the work, the entire drawing becomes conductive even when it dries out. This prevents the DT table from recognizing exactly where the current brush stroke is. We solved this problem by using Shodo red ink, which is not conductive.

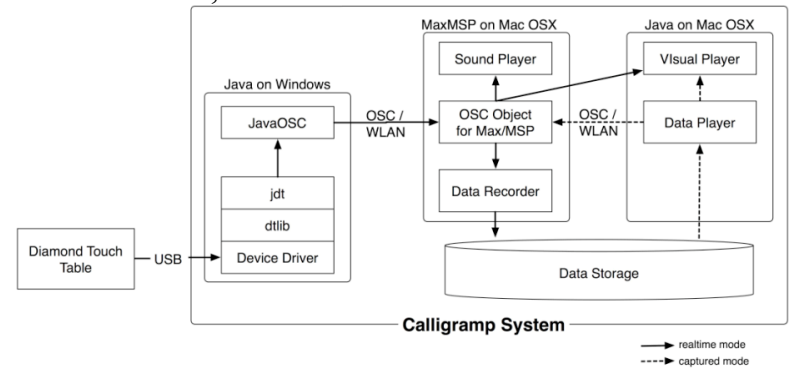

Figure 4: The Architecture of the Calligramp System

Figure 4 illustrates the architecture of the Calligramp system. The system supports two interaction modes: realtime mode, and capture mode. In the realtime mode, the brush strokes detected by DT are processed in realtime and visual and/or audio feedback is provided to the user. In the capture mode, the brush strokes detected by DT are collected for later replay. The solid line in Figure 4 represents the data flow in the realtime mode and the dotted line represents that of the capture mode.

\subsection{Data Collection}

We use DT SDK2.0, dtlib and jdt to access sensory data detected by the DT table on Windows XP. We first filter the data captured by the DT table, and then use JavaOSC to convert it into the OSC protocol and transfer it via wireless network to the MaxMSP program that runs on Mac OS X. The received data is then stored for replay. The data is currently stored as a non-proprietary text file.

We use two data formats, one for the realtime mode and the other for the capture mode. For the realtime mode, in order to minimize delay, we use the minimum set of information (see Figure 5(a)). The capture mode includes all realtime data plus calculated information containing more characteristics of the brush tuft movements (see Figure 5(b)).

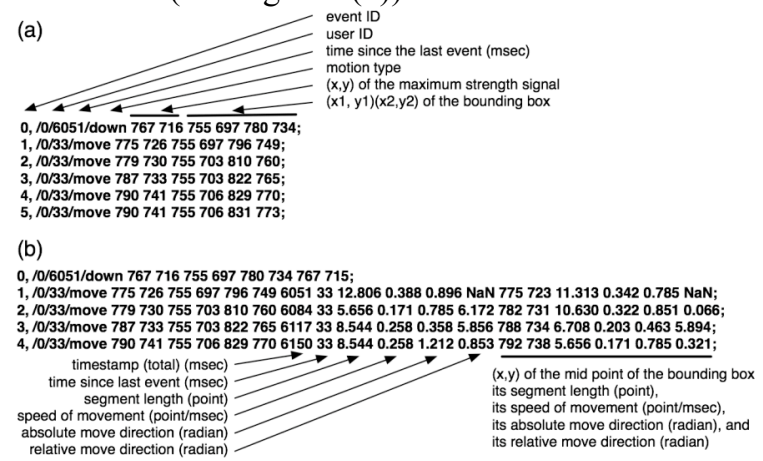

Figure 5: Data Formats Used in Calligramp

\subsection{Writing Processes}

To illustrate how a Shodo master and non-masters' writing processes differ, we have used the captured data to analyze the dynamics of stroke data produced by a Shodo master and non-masters.

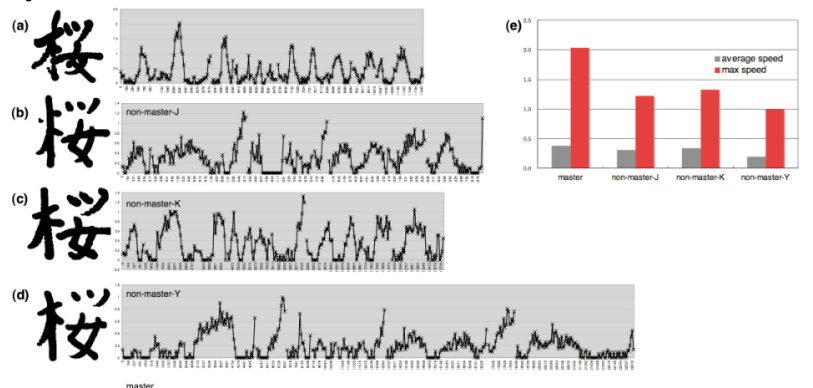

Figure 6: Process Differences between Shodo Master and Non-Masters

Figure 6(a) shows the data captured from a Shodo master and by three non-masters (Figure 6(b)-(d)), each with a graph showing how the stroke speed changes over time during the writing process. From the four graphs, one can see how rhythmical the master 
moves the brush by alternating high speed and low speed.

Figure 6(e) shows a graph comparing the average and maximum speed of moving a brush tuft by each of the four individuals. The master's (leftmost) graph has the highest value for speed.

\subsection{Feedback Mechanisms}

The Calligramp system currently supports two types of interaction with the augmented Shodo writing process: (1) after writing when the system reproduces visual effects and/or the theme music for the produced calligraphy, and (2) while writing when the system provides immediate visual and/or audio feedback or the system provides guidance for a writer by replaying the data captured from the writing of a Shodo master.

\subsubsection{The Visual Data Player}

The Visual Data Player component currently provides a movie player-like interface for interacting with the recorded stroke data. Figure 7 illustrates the visually reconstructed representation from the data. Figure 7(a) is a picture taken for the actual calligraphy written by a Shodo master. Figures 7(b)-(d) are screen shots of the Visual Data Player when loading the master's writing data.

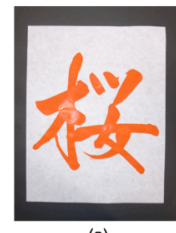

(a)



(b)

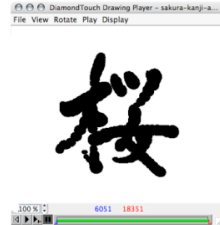

(c)

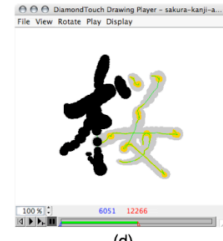

Figure 7: Real Writing (a) and Screen Shots of the Visual Player Component (b-d)

The Visual Data Player draws brush strokes as inscribed ovals along the path of the captured event data (Figure 7(b)). A series of the ovals colored in black produces the brush stroke like representation (Figure 7(c)).

In addition to a brush tuft's touches, the Visual Data Player component displays the peak lines (in green lines in Figure 7(d)) of the strokes by connecting line segments between each pair of two consecutive peaks (i.e., the max_signal_points). The yellow lines are peak lines using width as indicator of the inverse of the speed; the slower, the thicker (Figure 7(d)). A user may play back the writing process by using the movie player-like control bar located in the bottom of the window with various speeds (by changing the value in the window located in the bottom left; the default is $100 \%$ )

The Visual Data Player also provides mechanisms to view the writing from different angles as a way to augment the Shodo experience. For instance, Figure 8 shows the directed Tuft view mode, which shows the visual representation as if one becomes the tip of the tuft. The stroke always moves upward and the character instead moves around in the canvas area.

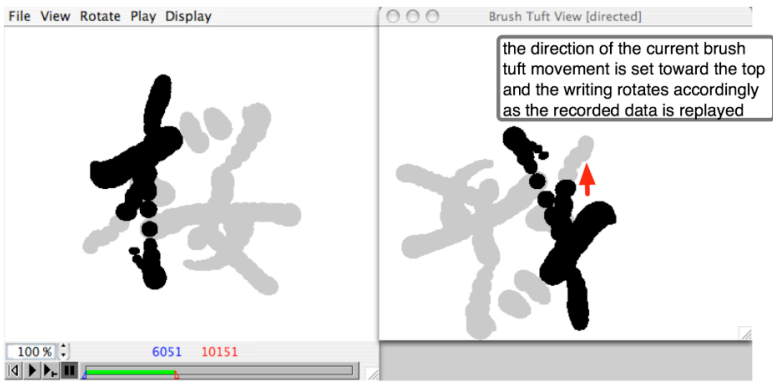

Figure 8: Different Views in the Visual Player

\subsubsection{The Sound Data Player}

To represent the rhythm and dynamism of the writing process, auditory display [5] is an effective way to emphasize such properties especially as the writing process is replayed.

We have implemented the Sound Data Player component by using MaxMSP to produce different kinds of auditory feedback for the data captured by the Calligramp system (Figure 9). We have particularly paid attention to designing sound representations that stress the rhythm and dynamism of the speed and changing direction of the brush tuft movement. We have currently implemented 26 sound primitives that can be linked to some of the properties of the event data entry as described in Figure 5.

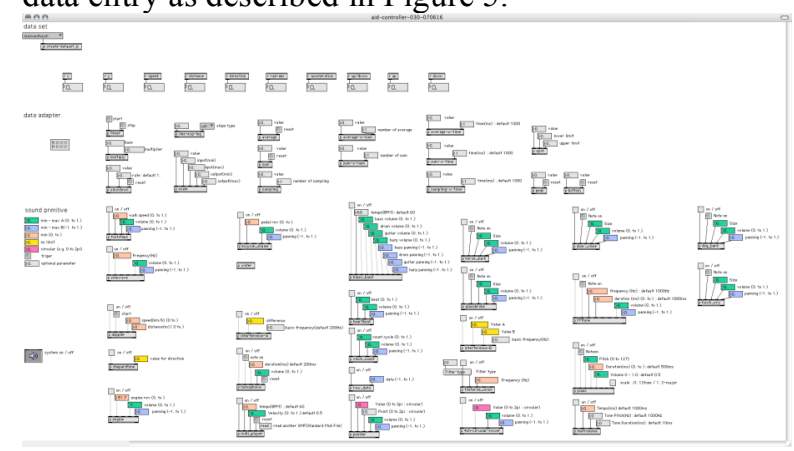

Figure 9: The Sound Data Player

For instance, one of the sound primitives we have implemented to stress the dynamism of the changing speed of the brush tuft, is the one that produces the sound of a racing car engine. It produces the enginelike sound with high or low RPM depending on how large or small the value is.

Examples of other sound primitives we have implemented include the metronome, footsteps, glass crush, piano scale, controlling music playing speed, and sinewave. We leave the description of details of each sound implementation to another paper due to the page limitation. 


\section{Discussions}

Many of the existing system support approaches for oriental calligraphy and brush drawing (such as [2] and [3]) simulate brushwork to produce visual representations of brush strokes. Our approach, in contrast, is to use the real brush, ink and a sheet of paper, to nurture the calligraphy experience in a natural setting. The visual representation exists as a real document, and we use the captured process data to augment the experience without ruining the real, natural, experience of writing.

The Calligramp system can be viewed as a history tool for writing, which enables people to re-experience the process of creating artifacts. Our underlying belief is that knowing the process of creation makes the difference in understanding the artifact. For example, the movie, "The Mystery of Picasso" [6], shows the painter's painting process, where most of paint moves made on the canvas had been overdrawn by later painting moves and did not remain in the final drawing. In the process, a variety of objects, characters and themes emerged, and disappeared by being overdrawn, literally embedded in the final drawing. One sees very different things in the resulted paining after one watches the movie and knows the process. Another example that shares the same philosophy is the airconditionvideo project [1]. An interesting abstract picture is made by taking a series of successive snapshots of a person's body movement. One cannot tell how the picture is created without looking at the process of making the movie. Once one understands the process, one can appreciate the picture in a completely different manner.

Shodo has a similar nature in its visual representation. When a Shodo master looks at a work of calligraphy, the master is able to reinterpret and reexperience the process from the resulted visual representation; that is, the dynamics and rhythm of the brush movement. For non-masters, one can only focus on the shape and visual attributes of the calligraphy. It is difficult to "read" from the visual representation the temporal and rhythmic aspects of the calligraphy. The goal of Calligramp is to partially make such temporal and rhythmic information available for those who are not Shodo masters.

The Calligramp project is still ongoing and can be expanded in several ways. First, we are still exploring the space of visual and sound design for better representing and communicating the writing process data. Once designed, visual and sound design alternatives need to be studied in order to understand the effectiveness of them as feedback. Second, we also plan to identify richer events from the data to better understand the writing process. For instance, we can identify the moment when two strokes intersect. We can then generate visual and/or audio emphases to the user, which gives innovative ways to experience Japanese calligraphy.

The project described in this paper is a way to augment a regular tabletop interaction experience in the form of Japanese calligraphy. Our design is not to reform the writing experience by itself. A user engages in Japanese calligraphy in a very traditional form by using a brush, ink, on a sheet of paper on the horizontal, flat surface. We use the captured data to augment the process.

We believe that our approach, which differs from most of existing Tabletop computing tools [6] since users are not directly interacting with computing objects, demonstrates a new direction in expanding the horizon of the tabletop computing.

\section{Acknowledgements}

We are very grateful to the Shodo Master, Kousei Yamane, for letting us observe and collect data from his precious Shodo performances. We would also like to thank MERL for generously letting us use a DT, and Brent Reeves and Yoshinari Shirai for valuable discussions. This work is supported by MEXT Grantin-Aid for Scientific Research (A) 16200008, 20042007.

\section{References}

[1] Aircondition, http://oliverlaric.com/airconditionvideo.htm

[2] W. Baxter, V. Scheib, M. Lin, and D. Manocha, DAB: Interactive Haptic Painting with 3D Virtual Brushes, Proceedings of ACM SIGGRAPH 01, pp. 461-468, 2001.

[3] N.S.H. Chu, C-L. Tai, Real-Time Painting with an Expressive Virtual Chinese Brush, IEEE Computer Graphics and Applications, pp.76-85, IEEE, September/October 2004.

[4] P.H. Dietz, D.L. Leigh, DiamondTouch: A MultiUser Touch Technology, ACM Symposium on User Interface Software and Technology (UIST), pp. 219226, November, 2001.

[5] G. Kramer, An Introduction to Auditory Display, Auditory Display: Sonification, Audification, and Auditory Interfaces, G. Kramer (Ed.), Addison-Wesley, Reading, MA., pp.1-77, 1994.

[6] The Mystery of Picasso, http://www.imdb.com/title/tt0049531/

[7] E. Takase, Learn Japanese Calligraphy, Takase Studios Inc., 2003. 